refugees themselves in all of these activities is essential. ${ }^{11}$ Such a response by the health service will require intersectoral coordination, extra funding, and, above all, commitment. In today's economic and political climate none of this is easy, but ignoring the refugee crisis will not make it go away.

GHADA KARMI

Consultant in Public Health Medicine,

Health and Ethnicity Programme,

North West and North East Thames Regional Health Authorities,

London W2 3QR

1 US Committee for Refugees. World refugee survey - 1989 in review: Washington, DC: US Committee for Refugees, 1990:30-4.
2 Home Office Statistical Department. Refugee Statistics, UK, Issue 22/90. London: HMSO (in press

Karmi G, ed. Refugees and the National Health Service. London: Health and Ethnicity Programme, North West and North East Thames Regional Health Authorities, 1992.

Refugee Policy Group. Ensuring the health of refugees: taking a broader vision. Washington: Refugee Policy Group, 1990.

Dick B. Diseases of refugees-causes, effects, and control. Trans $R$ Soc Trop Med Hyg 1984;78:734-41.

6 Bonneriea L. Shaming the world. The needs of women refugees. London: Change and World University Services, 1985.

British Refugee Council. Settling

British Refugee Council, 1987.

87.

Holtzman WH, Bornemann TH, eds. Mental health of immigrants and refugees. Austin, Texas: Hogg Foundation for Mental Health, University of Texas, 1990

9 Baker R. ed. The psychosocial problems of refugees. London: British Refugee Council and European Consultation on Refugees and Exiles, 1983:14-21.

10 National Institute of Mental Health. Mental health services for refugees. Washington, DC: US Department of Health and Human Services, 1991:205-18.

11 Dick B, Simmonds S. Primary health care with refugees: between the idea and the reality. Trop Doct $1985 ; 15: 2-7$

\title{
Controlling leprosy
}

\section{Multidrug treatment is not enough alone}

A paper in this week's journal on the ocular complications of leprosy in a Romanian leprosarium reminds us of the appreciable morbidity of the disease-much of it avoidable (p 240). Will the World Health Assembly's goal of eliminating leprosy as a public health problem by 2000 soon make this paper of little more than historical interest?

The past decade has witnessed the most dramatic changes in the control of leprosy since dapsone was introduced 40 years ago. In particular, the introduction of short course multidrug treatment (with rifampicin, dapsone, and clofazimine) has had far reaching effects on the structure and strategy of leprosy control programmes. ${ }^{2}$ In 1985 the World Health Organisation formulated three main objectives for controlling leprosy. These were to interrupt transmission of infection; to cure patients and, where possible, fully rehabilitate them; and to prevent deformities from developing. ${ }^{3}$ Multidrug treatment has since been promoted as the main tool to achieve these objectives. Though undoubtedly an advance, this emphatic promotion overlooks other important trends and influences in the control of leprosy.

Short course multidrug treatment has led to a dramatic fall in caseload in countries where leprosy is endemic because large numbers of patients have been discharged who would previously have remained on treatment registers for many years, if not for life..$^{+6}$ It is important to recognise that these falls in "prevalence" do not themselves indicate falls in incidence. Indeed, no data are yet available to show that treatment of cases of leprosy reduces incidence.

On the other hand (and accepting caveats that observed trends may reflect changes in case finding and case definition), dramatic falls in the incidence of leprosy (or case detection rates) have been documented in many countries for more than a decade. ${ }^{7}$ As these falls began in most countries long before the introduction of multidrug treatment at least two other factors should be considered. Firstly, much evidence exists that the incidence of leprosy falls with improving socioeconomic standards, ${ }^{8}$ and the recent falls in incidence are likely to be related, at least in part, to these important, though ill defined, influences. Secondly, BCG vaccination has consistently been shown to protect against leprosy, ${ }^{9}$ and recent investigations have shown that it imparts greater protection against leprosy than against tuberculosis. ${ }^{10}$ Furthermore, studies suggest that BCG vaccine imparts protection against multibacillary ("many bacilli," lepromatous) as well as against paucibacillary ("few bacilli," tuberculoid) disease. ${ }^{10} 11$ This is important in so far as cases of multibacillary disease act as sources of infection and their prevention should thus reduce transmission and ultimately the overall incidence of disease in the community. ${ }^{8}$

Current evidence shows that multidrug treatment is highly effective in reducing bacillary load and thereby ridding 은 patients of Mycobacterium leprae. ${ }^{12}$ Furthermore, the short- $N$ ened course and the requirements for supervising the monthly doses of rifampicin have greatly reduced the problem of compliance. Patients with leprosy may still, however, develop disability due to nerve damage during or after chemotherapy. ! Because of the early discharge of patients systematic surveillance after treatment has been recommended to detect early signs of reaction and relapse. ${ }^{13}$ Various studies have shown that, in patients with paucibacillary disease, cellular hypersensitivity (type 1) reactions occur mainly within the first year after the end of treatment, whereas relapses are more likely to occur three to four years later. ${ }^{1+15}$ Reactions affect mainly nerves of the hands, feet, and face-thus resulting in disabilities, ${ }^{16}{ }^{17}$ which are preventable if treated early. ${ }^{15}$ Corticosteroids have long been used to treat reactions, and there is current interest in improving these regimens and in using cyclosporins and similar drugs. ${ }^{18}$

The World Health Assembly's resolution defined elimination as a prevalence of under one case per 10000 population. Prevalence, however, is not the only indicator important for public health. Without a reduction in incidence the problems of case finding, diagnosis, and registration will remain. And if disabilities are not reduced the disease's burden will remain. For these reasons multidrug treatment should not be regarded as the sole agent for controlling leprosy. This is particularly important in so far as recent data suggest a slight fall in the proportion of people with leprosy worldwide currently taking this treatment, reflecting the logistic problems of extending the policy to places that are difficult to reach. ${ }^{19} \mathrm{~A}$ possible $\stackrel{\oplus}{-}$ solution is to shorten regimens still further. The recent 0 finding that ofloxacin is bactericidal against $M$ leprae has $\frac{\vec{D}}{\mathbb{D}}$

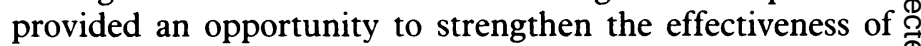
multidrug treatment and further reduce its duration..$^{20} \stackrel{\mathbb{Q}}{\mathbb{2}}$ Clinical trials of an ultrashort regimen based on a one month course of ofloxacin have recently been initiated by. WHO, but several years will elapse before their results become available.

For now, it seems that, whatever its duration, multidrug? treatment is relevant mainly to achieving the second of WHO's objectives for leprosy control programmes - that is, curing and rehabilitating patients. According to recent results 
from Venezuela and Malawi, such programmes might also contribute to the first objective-interrupting transmission by promoting BCG vaccination or revaccination of contacts. ${ }^{21}$ Achieving the third objective-preventing deformitiesrelies mainly on surveillance to ensure early detection of nerve damage and appropriate treatment for reactions. Until the first two objectives are achieved the paper by ffytche and colleagues will remain relevant.'

CHRISTIAN LIENHARDT Lecturer

P E M FINE

Reader

Communicable Disease Epidemiology Unit,

London School of Hygiene and Tropical Medicine,

London WC1E 7HT

1 ffytche TJ, Brandt F, Cerchez J, Stoicescu S. Stingl P, Filitis M. Ocular complications observed in leprosy patients in Romania. BMF 1992;305:240-2.

2 World Health Organisation Study Group. Chemotherapy of leprosy for control programmes. WHO Tech Rep Ser 1982; No 675 .

3 World Health Organisation Study Group. Epidemiology of lepross in relation to control. WHO Tech Rep Ser 1985; No 716.

4 Nordeen SK, Lopez Bravo L, Daumerie D. Global review of multidrug therapy (MDT) in leprosy World Health Stat $Q$ 1991:44:1-15.
5 Jesudasan K, Vijayakumaran P, Pannikar VK, Christian M. Impact of MDT on leprosy as measured by selective indicators. Lepr Rev 1988:59:215-23.

6 Revankar CR, Pawar PL, Belurkar LS, Pai RR, Ganapati R. Reduction in case-load after MDT in an uran leprosy control programme. A retrospective study in Bombay. Lepr Rev 1991:62:44-8. an Ura Reflon 7 Fine PEM. Reflections on the elimination of leprosy. Int f Lepr Other Mycobact Dis 1992;60:71-9.

8 Fine PEM. Leprosy - the epidemiology of a slow bacterium. Epidemiol Rev 198

9 Fine PEM, Rodrigues LC. Mycobacterial vaccines. Lancet 1990;335:1016-20.

onnighaus JR, Fine PEM, Sterne JAC, Wilson R, Msosa E, Gruer P, et al. Efficacy of BCG vaccine against leprosy and tuberculosis in northern Malawi. Lancet 1992;339:636-9.

11 Muliyil J, Nelson KF, Diamond EL, Effect of BCG on the risk of leprosy in an endemic area: case control study. Int $\mathcal{f}$ Lepro Other Mycobact Dis 1991;59:229-36.

12 Grosset JH. Recent advance in the chemotherapy of leprosy. Lepr Rev 1990;61:313-29.

3 Becx-Bleuminck M. Operational aspects of multidrug therapy. Int 7 Lepr Other Mycobact Dis 1989;57:540-51

14 Waters MFR, Ridley DS, Ridley MJ. Clinical problems in the initiation and assessment of multidrug therapy. Lepr Rev 1986;57(suppl):92-100.

15 Boerrigter G, Ponnighaus JM, Fine PEM, Wilson RJ. Four year follow-up results of a WHO recommended multiple drug regimen in paucibacillary leprosy patients in Malawi. Int $\mathcal{f}$ Lepr Other Mycobact Dis 1991;59:255-61.

16 Becx-Bleuminck M. The management of nerve damage in the leprosy control services. Lepr Rev 1990;61:1-11

17 Rose P, Waters MFR. Reversal reactions in leprosy and their management. Lepr Rev 1991:62:113-9.

18 Frankel RI, Mita RTR, Dann FJ. Resolution of type 1 reaction in multibacillary Hansen's diseases as a result of treatment with cyclosporin. Int I Lepr Other Mycobact Dis 1991;60:8-12.

19 Gelin C. Declerc E. Global evaluation of the introduction of multidrug therapy, Leprosy Epidemiological Bulletin 1992;8:1-44.

20 Grosset JH, Ji B, Guelpa-Lauras CC, Perani EG, Nideli LN. Clinical trial of pefloxacin and ofloxacin in the treatment of lepromatous leprosy. Int $f$ Lepr Other Mycobact Dis 1990;58:281-5. 21 Convit J, Sampson C, Zuniga M, Smith PG, Plata J, Silva J, et al. Immunoprophylactic trial with combined Mycobacterium leprae/BCG vaccine against leprosy: preliminary results. Lancet 1992;339:446-50.

\section{Saliva as a diagnostic fluid}

\section{Second now to blood?}

Interest has been increasing in non-invasive diagnostic testing. Some of this attention stems from the AIDS epidemic, which has provided a new rationale for haemophobia, while other factors include new developments in home based diagnostic tests, a demand for samples collected in the home or workplace, and the close linkage of biotechnology to diagnostic tests.

Diagnostic tests based on fluid generally use blood and urine and less frequently the esoteric fluids such as saliva, sweat, and tears. Saliva's popularity has suffered because it lacks "the drama of blood, the sincerity of sweat and the emotional appeal of tears." Sweat and tears, however, are difficult to obtain in sufficient quantities for routine testing, and urine will always lack the charisma of the other fluids. Saliva, by default, therefore becomes the most favoured alternative to blood.

Over 2500 papers dealing with salivary diagnostic tests have been published since 1983, and an extensive bibliography is being prepared for a forthcoming meeting on the topic, sponsored by the New York Academy of Sciences, to be held on 22-25 October in Florida. A detailed analysis of these papers is under way, but several observations are already clear. The sources of the publications are wide and diverse in terms of both country of origin and topic. Subjects range from forensic medicine to clinical endocrinology and from dental medicine to veterinary science. Increasingly, saliva is being used to monitor antibodies (to viruses ${ }^{23}$ and bacteria ${ }^{+5}$ ), drugs of misuse, ${ }^{67}$ and steroid hormones. ${ }^{89}$ Saliva's main advantage is that the patient can collect samples at home when clinically relevant or in other places, including the workplace, where collecting blood or urine may be difficult.

Most molecules present in blood or urine can also be detected in salivary secretions. Their concentrations in saliva are usually one tenth to one thousandth of those in blood. Although highly sensitive methods of detection are required, technical advances have made this feasible. Studies of the correlation between concentrations in blood and saliva have found examples of excellent concordance (ethanol, cortisol, theophylline, and antibodies to HIV) and poor concordance (thyroxine, dihydroepiandrosterone, prolactin, and adrenocorticotrophic hormone). ${ }^{10}$

Ideally, the salivary concentration of the drug or hormone should be independent of the salivary flow rate and should correlate with blood concentrations of the substance. An important question is how drugs and hormones enter saliva. Conjugated steroids seem to enter through tight junctions in a flow related process (hormone concentration increases as salivary flow rate increases), and their concentrations in saliva are therefore unlikely to provide a useful measure of their concentrations in blood.

Finally, how saliva is collected is important. Many doctors, comfortable with collecting blood and urine, are intimidated by the idea of collecting saliva. Aesthetic and scientific considerations arise, including which fluid is being collected (whether duct saliva, whole saliva, or gingival crevicular fluid) and the method of recovering molecules from the collecting device. Gingival crevicular fluid is a serum transudate present in the mouth that often more closely resemble serum, but obtaining it in sufficient quantities is difficult. Several companies have devised oral collection devices - for example, a sac for small molecules, an immobilised support, and a device for collecting an oral transudate.

Tests based on saliva have already made substantial inroads into diagnosis. For some molecules - for example, antibodies, unconjugated steroid hormones, and certain drugs - the techniques are sufficiently sensitive to reflect blood concentrations of the substance accurately. New collecting devices should make doctors more comfortable with using saliva as an alternative to blood. Future research should define further the molecules, patients, and circumstances best suited to this form of testing.

Professor of Biochemistry,

DANIEL MALAMUD

School of Dental Medicine,

Philadelphia,

PA 19104, USA 